\title{
Wildwestmanieren im Gesundheitswesen?
}

SW!SS REHA-Vorstand

Korrespondenz:

SW!SS REHA

Laurenzenvorstadt 77

Postfach

CH-5001 Aarau

Tel. 0628245152

Fax 0628225581

info@swiss-reha.com

www.swiss-reha.com
Die klassische Szene aus alten Wildwestfilmen ist bekannt: Gesetz und Ordnung sind nur ein belangloses Stück Papier. Es gilt das Recht des Stärkeren: Wer zuerst schiesst, hat gewonnen...

Zum Glück hat unsere zivilisierte Gesellschaft diese archaischen Verhältnisse hinter sich gelassen - sollte man jedenfalls meinen. Für das Gesundheitswesen scheint das jedoch nicht zu gelten. Denn verschiedene Krankenversicherer brechen offensichtlich und bewusst geltendes Recht. Der zuständige Bundesrat duldet, toleriert und fördert das illegale Treiben und versucht, es im nachhinein gesetzlich zu legitimieren.

Die Folgen sind absehbar: Auf der Strecke bleibt dabei zum einen das Wohl des «kleinen Mannes», der Patientinnen und Patienten, zum anderen die Rechts- und Planungssicherheit im Gesundheitssystem und - in der Konsequenz die bislang hohe Qualität des schweizerischen Gesundheitswesens.

\section{Doch zuerst die Fakten}

1. Das geltende Krankenversicherungsgesetz untersagt es Krankenversicherern - ausser im Notfall - im Ausland erbrachte medizinische Leistungen für Grundversicherte zu übernehmen (Territorialprinzip).

2. Die Krankenkassen Helsana und CSS verkünden seit Jahresbeginn, dass sie auch Patienten aus der Grundversicherung ins Ausland zur Behandlung schicken.

3. In der NZZ (10. November 2005) erklärt Bundesrat Couchepin im Hinblick auf die Aktivitäten der Versicherer, dass er «nicht Polizist spielen» werde und das Bundesamt für Gesundheit angewiesen habe, das geltende Gesetz grosszügig anzuwenden.

4. In seiner Antwort auf eine Interpellation von Ständerat Hermann Bürgi zur «Übernahme der Kosten von medizinischen Behandlungen im Ausland» erklärt Bundesrat Couchepin am 23. November 2005: «Aufgrund des Territorialitätsprinzips ist es den Krankenversicherern grundsätzlich nicht gestattet, im Ausland erbrachte Leistungen im Rahmen der obligatorischen Krankenpflegeversicherung zu übernehmen. [...] Das Bundesamt für Gesundheit interveniert in seiner Funktion als Aufsichtsbehörde, falls ein Krankenversicherer die gesetzlichen Bestimmungen nicht einhält.»

5. Obwohl das geltende Gesetz eine Behandlung von Patienten aus der Grundversicherung verbietet, vereinbart Bundesrat Pascal Couchepin am 21. Dezember 2005 mit dem zuständigen Minister von Baden-Württemberg ein dreijähriges Pilotprojekt über die grenzüberschreitende Zusammenarbeit beider Basel mit Süddeutschland im Bereich der Gesundheitsleistungen auch für Grundversicherte.

6. Um sein gesetzwidriges Handeln nachträglich zu legitimieren, eröffnet das Eidgenössische Departement des Inneren am 12. Januar 2006 die Vernehmlassung zur Änderung des Krankenversicherungsgesetzes. Die Änderung soll das EDI dazu ermächtigen, zeitlich begrenzte Projekte zu bewilligen, die die Übernahme von gewissen im Ausland erbrachten medizinischen Leistungen durch die Krankenkasse vorsehen. Das Gesetz soll am 1. Mai 2006 in Kraft treten.

7. Während der Frühjahrssession der eidgenössischen Räte kommt es am 8. März 2006 im Rahmen der Teilrevision über das Bundesgesetz über die Krankenversicherung bei der Debatte über die Spitalfinanzierung im Ständerat zu einem Wortgefecht über den Einkauf von Spitalleistungen im Ausland. Bundesrat Pascal Couchepin wird für sein Verhalten gegenüber dem offensichtlichen Rechtsbruch verschiedener Krankenversicherungen gerügt. Mit seiner Politik werden die kantonalen Spital- und Bettenplanungen und damit Investitionen in Milliardenhöhe gefährdet. Zudem wird der Bundesrat dafür kritisiert, dass die Krankenkassen mit bundesrätlicher Duldung geltendes Gesetz «kalt» aushebeln.

\section{Worum geht es?}

Mittelpunkt jeglichen medizinischen Handelns ist das Patientenwohl - seine bestmögliche Versorgung, Heilung, Therapie und Wiedereingliederung. Das Schweizer Gesundheitswesen und im besonderen die Schweizer Rehabilitationsmedizin sind weltweit führend. Zudem wurde im März 2006 an einer nationalen Fachtagung in Luzern übereinstimmend festgehalten, dass Spitzenmedizin ohne gleichwertige Rehabilitation in der Schweiz nicht möglich ist.

Eine kürzlich publizierte Studie zeigt, dass im Vergleich zu einer Klinik in den USA die Rehabilitation von Schlaganfallpatienten in einer Schweizer Klinik zu besseren Resultaten der Funktionsfähigkeit und der Unabhängigkeit führt. Sie bestätigt damit die Erkenntnisse einer früheren Studie (Interregio-Studie), die Spitäler im Dreiländereck einem Qualitätsvergleich unterzogen hat. 
Die Schweizer Rehabilitationsmedizin leistet damit einen wichtigen Beitrag zur Volksgesundheit sowie zur volkswirtschaftlichen Produktivität. Denn jede erfolgreiche nachhaltige Therapie erübrigt kostenintensive Nach- und Folgebehandlungen. Davon profitiert die ganze Volkswirtschaft.

Verschiedene Krankenversicherer verfrachten nun Schweizer Patienten vor allem ins süddeutsche Ausland. Dies mit der Begründung, dort seien die Qualität und der Komfort höher, die Qualitätsnormen transparenter und die Preise tiefer. Dazu sei festgehalten:

1. In der Schweiz wird die Ergebnisqualität von Rehabilitationskliniken mit wissenschaftlich anerkannten Tools bereits seit Jahren repräsentativ gemessen. Der Einsatz solcher Instrumente ist zum Teil schon in Tarifvereinbarungen zwischen Versicherern und Spitälern eingeflossen.

2. Der Kostenvergleich zwischen süddeutschen und Schweizer Kliniken vergleicht Äpfel mit Birnen. Denn in den ausländischen Kliniken werden vor allem leichte Fälle behandelt. Die schweren und damit betreuungs- und kostenintensiven bleiben weiterhin in der Schweiz.

3. Süddeutsche Kliniken - viele von ihnen stehen am Rande des Konkurses oder sind hochgradig defizitär - können mit Dumpingpreisen aufwarten, da ihnen der Staat die Defizite deckt. Dies im Gegensatz zur Schweiz, wo die RehaKliniken kostendeckend arbeiten müssen.

4. Ein Klinikaufenthalt zur Rehabilitation ist kein Kuraufenthalt. Die Qualität in der Rehabilitation darf nicht mit Marmorfussböden oder Designermöbeln in den Zimmern verwechselt oder gleichgestellt werden. Zufriedenheitsmessungen in Schweizer Kliniken zeigen, dass sie auch in diesem Bereich keinen Vergleich zu scheuen haben.

5. Die deutschen Rehakliniken beschäftigen pro 100 Betten viel weniger Klinikpersonal, was die Behandlung von schwerkranken Patienten nicht zulässt und den Preisvergleich massiv verzerrt.

6. Geht es um die Gesundheit von Menschen, ist Preis- und Zeitdruck ein denkbar schlechtes Mittel. Dünne Personaldecken und Zeitdruck sind schlechte Voraussetzungen für medizinische Qualitätsarbeit! Damit wird die Gesundheit von Patienten fahrlässig aufs Spiel gesetzt.

\section{Die Politik von Bundesrat Couchepin und den Versicherern}

Trotz dieser offenkundigen Faktenlage hat Bundesrat Couchepin entschieden, eine Teilrevision der Verordnung vom 27. Juni 1995 über die Krankenversicherung in die Vernehmlassung zu schicken. Dies mit dem Ziel, ein grenzüberschreitendes Pilotprojekt der Kantone BaselStadt, Basel-Landschaft und des Landkreises Lörrach zu legitimieren. Der Bundesrat soll im nachhinein das gesetzeswidrige Verhalten der Krankenkassen sanktionieren.

Worum es den Versicherern geht, liegt auf der Hand: Sie wollen sich mit dem Export Schweizer Patienten ins Ausland gesundstossen. Denn würde es den Versicherern um einen wirklich freien und fairen Wettbewerb gehen, würden sie sich auch nicht dagegen wehren, den Schweizer Markt für günstigere ausländische Versicherer zu öffnen.

\section{Die Position von SW!SS REHA}

SW!SS REHA, der Verband der schweizerischen Rehabilitationskliniken, erwartet keinen Heimatschutz und stellt sich dem Wettbewerb - sofern für alle Marktpartner die gleichen Voraussetzungen gegeben sind. Deshalb müssen in einem ersten Schritt die innerschweizerischen Barrieren fallen, denn aktuell ist aufgrund der kantonalen Spitalplanungen und Spitallisten eine freie Spitalwahl nicht möglich. Ebenso muss die Ergebnisqualität mit einem einheitlichen System über die Landesgrenzen hinweg gemessen werden. Es geht auch nicht an, dass den Schweizer Rehabilitationskliniken nur die schwerst rehabilitationsbedürftigen Fälle zugewiesen werden, während die einfacheren Fälle ins Ausland verwiesen werden. Da heute die Diskussion von den Krankenkassen nur auf ökonomischer Ebene und nicht mit Blick auf das Patientenwohl geführt wird, erstaunt es um so mehr, dass die Versicherer zum Beispiel einer Differenzierung der stationären Tagespauschalen für die muskuloskelettale Rehabilitation in Aargauer Reha-Kliniken für leichte, mittlere und schwere Fälle nicht zugestimmt haben.

In diesem Zusammenhang fordert SW!SS REHA die Einhaltung von fairen Wettbewerbsbedingungen. Dazu gehört die wissenschaftliche Auswertung des Pilotprojektes nach internationalen Standards, insbesondere auch der Langzeitwirkung. Nur nachhaltige Therapieerfolge führen zu den volkswirtschaftlich erwünschten Kosteneinsparungen. Zudem müssen alle Partner an der Auswertung des Pilotprojektes beteiligt sein, nur so lässt sich verhindern, dass es zu einer bestellten Auswertung im Sinne der Versicherer kommt. 